\title{
Co-simulation of a Tracked Mobile Robot Based on RecurDyn and Simulink
}

\author{
Jun $\operatorname{Han}^{1, a}$, Guoquan Ren ${ }^{1, b}$ and Dongwei $\mathrm{Li}^{1, \mathrm{c}}$ \\ ${ }^{1}$ Mechanical Engineering College, Shijiazhuang 050003,China. \\ ahanjun_wy@163.com, ${ }^{b} 709955574 @ q q . c o m,{ }^{c} 121 d w @ 163 . c o m$
}

Keywords: Tracked Mobile Robot, RecurDyn, Simulink, Co-simulation.

\begin{abstract}
In this paper, based on the multi-body dynamics simulation software, RecurDyn, virtual prototype model of a tracked mobile robot has been built, and with the RecurDyn interface connected to the Simulink control software, it was realized to build a control co-simulation platform of the tracked mobile robot. Through setting control conditions in Simulink, it was achieved to make the virtual prototype model tracking specific longitudinal motion velocity and turning velocity. The simulation results verified the accuracy and effectiveness of the co-simulation model, and the method is of great benefit to study motion tracked mobile robot control algorithm in the next step.
\end{abstract}

\section{Introduction}

Crawler type mobile robot is a kind of common mobile robot, because of its adaptability to ground crawler structure design is excellent, and is widely used in city rescue, EOD and submarine detection in complex environment [1-2]. In the research of the motion control of the tracked mobile robot, because of its complex mechanical system and changeable use environment, the traditional experience combined with the experimental method has a long research cycle and a large cost. With the development of virtual prototype technology and multi-body dynamics analysis software, researches on tracked mobile robot, through the establishment of a virtual prototype model of accurate, can use virtual tests under various conditions to reduce the cost of test, which has an important significance to shorten the development cycle [3-4].

\section{Virtual Prototype Modeling of TMR}

Virtual prototype analysis software RecurDyn, on the basis of multi-body system dynamics theory, the relative coordinate system equations and complete recursive algorithm, reduce the number of constraint equations of absolute coordinate system, suitable for [5] multibody system dynamics for solving large-scale complex problems. The high speed track system Track/HM kit, including driving wheel, wheel, wheel, induction roller, high speed track driving system and other components, can realize the precise modeling of vehicle action part. At the same time, the Ground module can be used to build various road models.

\subsection{Geometric Modeling and Assembly}

The virtual prototype model of the tracked mobile robot is composed of a car body and two crawler subsystems. The interface of CAD modeling of the virtual prototype model of the car body can be provided by RecurDyn can be established, first built vehicle model by SolidWorks, Pro/E and UG three-dimensional modeling software, and then establish a method into the ParaSolid format to RecurDyn. In this paper, the Pro/E software is used to build the tracked mobile robot ontology model, and then the RecurDyn method is used to build the vehicle model. The robot's two crawler subsystem is based on RecurDyn to provide high-speed track system toolkit Track /HM.

Each crawler system comprises 1 driving wheels, 1 Inducing wheels, 89 track plates and a suspension system composed of 4 damping springs, a plurality of suspension beams and a supporting frame of the 4 bearing wheels, which are composed of a plurality of loading wheels and a plurality of loading wheels. The track plate adopts single pin link, single tooth structure, the number of active teeth is 11 , and the effective radius is $\mathrm{r}=0.0677 \mathrm{~m}$. By calling the Track-HM 
module, according to the specific structure of each sub model of the robot's actual situation, determine the geometry parameters and the action part of all parts of the performance parameters, installation position and connection mode, finally assembled a virtual prototype model of the tracked mobile robot, as shown in Figure 1.

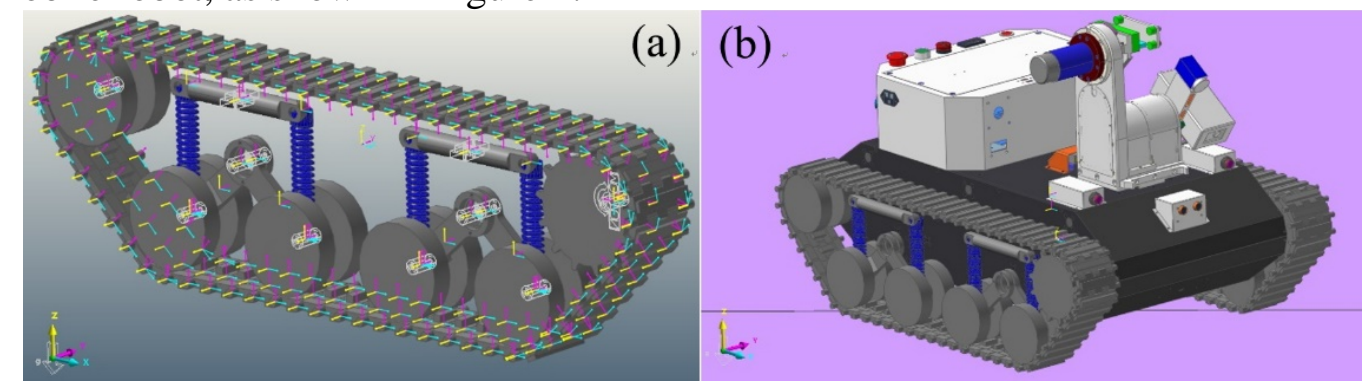

Fig.1 tracked mobile robot track subsystem (a) and virtual prototyping model (b)

\subsection{Add constraints and control input and output}

The constraint of each component of the mobile robot is set up, including structural constraints (contact constraints), spring force constraints, motion constraints and rigid body collision constraints. The applied constraints include: (1) driving wheel, wheel, wheel rotation induced by supporting constraint between frame and the vehicle body; (2) the fixed hinge constraint between suspension beam and the vehicle body; (3) wheel supporting a rotating wheel frame with side constraints; (4) damping spring force constraints. Wheel supporting frame with suspension beam; (5) the collision between the track and ground constraints. The constraint (2) (3) (4) constitutes the restraint of the suspension system of the tracked mobile robot, and the constraint (5) is automatically completed by the software.

You add constraints to be applied to the motion of tracked mobile robot driven set method for rotating the driving wheel in two pair of attributes in the shoe strap module check the motion movement constraints, the driving function set motion constraints for speed, with speed function value of PIN (w1). Similarly, the right track driving wheel speed can be set to PIN (w2). w1 and w2 expression by co-sim in the function area added, said co-simulation interface input value. And then set up the model output, generally based on the kinematics of motion control needs of several physical quantities are $\mathrm{x}, \mathrm{y}$, yaw, $\mathrm{w}$ and $\mathrm{v}$, the meaning and expression of the following table:

Table1 Plant Output Parameters

\begin{tabular}{ccc}
\hline parameters & expressions & meanings \\
\hline $\mathrm{x}$ & $\mathrm{x}=\mathrm{DX}(\mathrm{CM})$ & x-position of TMR \\
$\mathrm{y}$ & $\mathrm{y}=\mathrm{DY}(\mathrm{CM})$ & $\mathrm{y}$-position of TMR \\
yaw & $\mathrm{yaw}=\mathrm{YAW}(\mathrm{CM})$ & yaw of TMR \\
$\mathrm{w}$ & $\mathrm{w}=\mathrm{WZ}(\mathrm{CM})$ & turning velocity of TMR \\
$\mathrm{v}$ & $\mathrm{SQRT}\left[\mathrm{VX}(\mathrm{CM})^{\wedge} 2+\mathrm{VY}(\mathrm{CM})^{\wedge} 2\right]$ & longitudinal velocity of TMR \\
\hline
\end{tabular}

\subsection{Road Modeling}

In RecurDyn, the ground parameters can be defined in the crawler subsystem, and each track system can define the contact parameters between the pavement and track. According to the need can also be set as soft or hard road surface, this paper set up the simulation of the ground type for soft ground -clayey soil, the ground parameters as shown in figure 2: 


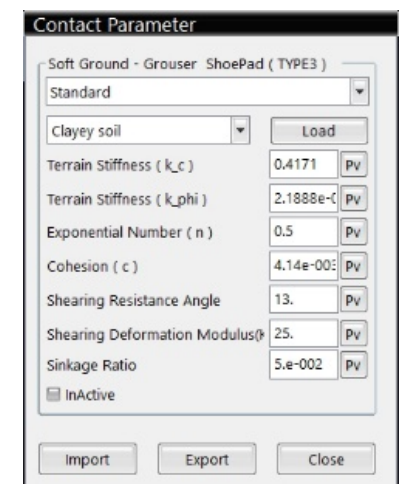

Fig.2 Ground Parameters

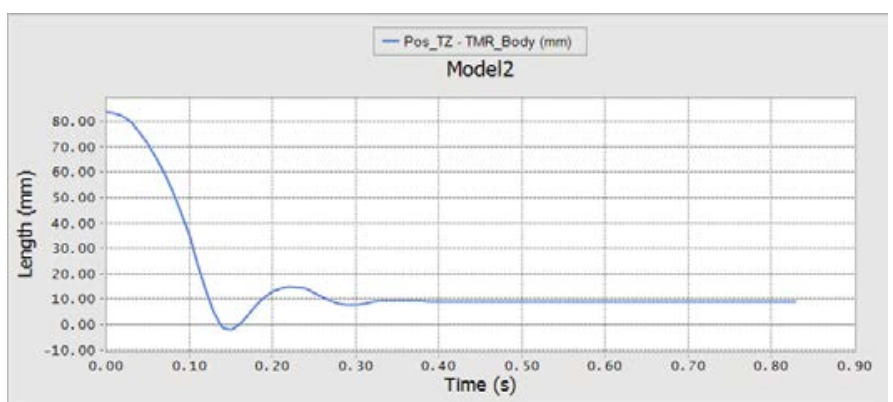

Fig.3 the vertical position of the centroid of robot

This virtual prototype model of the tracked mobile robot is established, in order to verify the effectiveness of the virtual prototype of the suspension, can set the tracked mobile robot from the free height from the ground $0.152 \mathrm{~m}$ down, down RecurDyn output to the robot centroid position change simulation results as shown in figure 3:

From here you can see that, then a robot rebound in about $0.15 \mathrm{~s}$, finally after about $0.4 \mathrm{~s}$ robot static, it can be seen that the virtual prototype model is correct, there are obvious suspension cushioning.

\section{Co-simulation Model of Tracked Mobile Robot}

RecurDyn software provides a general Simulink interface, the interface to generate a RecurDyn subsystem module in Simulink (as shown in Figure 4 (b)), Simulink controller according to the input and output of the subsystem module are connected, so as to realize the bidirectional transmission of data. The transfer process is: in a simulation time, Simulink will be set to the model input to the RecurDyn software, and RecurDyn software will be the virtual prototype simulation output to Simulink. 2 software to calculate the respective strengths of the next time step of the above variables, exchange again. So cycle until the end of the simulation [6].

The United simulation case in this set is produced by the demand of the tracked mobile robot Simulink longitudinal line speed and steering angular velocity signals generated by the robot speed distribution model of left and right side of the crawler driving wheel speed, the motor model generation of virtual prototype for RecurDyn speed input, finally by the RecurDyn simulation of virtual prototype of tracked mobile robot action the corresponding parameters, output feedback to Simulink. Control system Simulink connection Figure 4 (a):

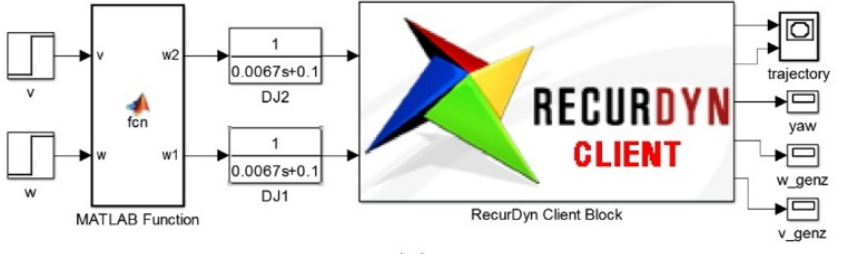

(a)

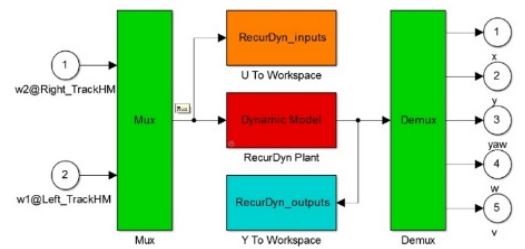

(b)

Fig.4 RecurDyn-Simulink co-simulation control system 
architecture (a) and RecurDyn module (b)

According to the longitudinal velocity and steering angle of the tracked mobile robot, the MATLAB Function model is used to calculate the speeds of the track wheel. A simple kinematic model is used to calculate:

$$
\left[\begin{array}{c}
\omega_{\mathrm{L}} \\
\omega_{\mathrm{R}}
\end{array}\right]=\frac{1}{r}\left[\begin{array}{cc}
1 & -B / 2 \\
1 & B / 2
\end{array}\right]\left[\begin{array}{l}
v \\
\omega
\end{array}\right]
$$

In the calculation formula above, $\omega_{L}$ is the rotate speed of the left track drive wheel, while $\omega_{R}$ is the rotate speed of the right track drive wheel. $r$ is the radius of the track drive wheels, here $r=0.0677 \mathrm{~m}$, and $B$ is distance between two tracks, according to the model size, $B=0.5075 \mathrm{~m}$. And $v$ is the longitudinal velocity, while $\omega$ is steering angular velocity of the tracked mobile robot.

The modular, DJ1 and DJ2 in the diagram, are expressed on the motor model, which drives the rotation of drive wheels of two tracks. The motor model is very complex, there we use transfer function $\frac{1}{0.0067 \mathrm{~s}+1}$ to characterize, which agrees with the speed curves of vehicle experiment well, only to express the delay characterization of motor tracking speeds.

\section{Co-simulation Experiment}

We set $v$ as a step signal, stepping from 0 to 0.05 when timer is $0.5 \mathrm{~s}$. And set $\omega$ as a step signal as well, stepping from 0 to 0.15 when timer is $2.5 \mathrm{~s}$. This to say, we control the tracked mobile robot moving from rest to longitudinal velocity $0.05 \mathrm{~m} / \mathrm{s}$ size at $0.5 \mathrm{~s}$, and then turning with the size of $0.15 \mathrm{rad} / \mathrm{s}$ steering angular velocity at $2.5 \mathrm{~s}$. The simulation results of the trajectory of the virtual prototype are shown in Figure 5. And the simulation results of the longitudinal velocity tracking are shown in Figure 6. Finally, the tracking results of the steering angular velocity are shown in figure 7.

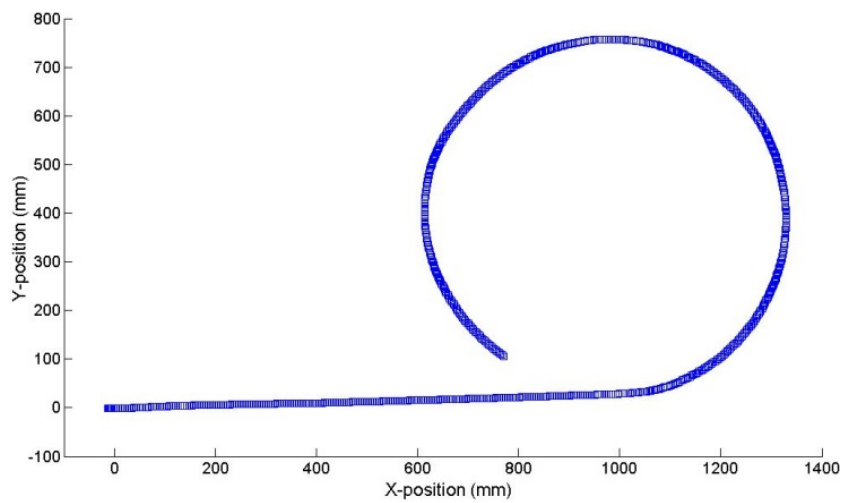

Fig.5 Trajectory Map

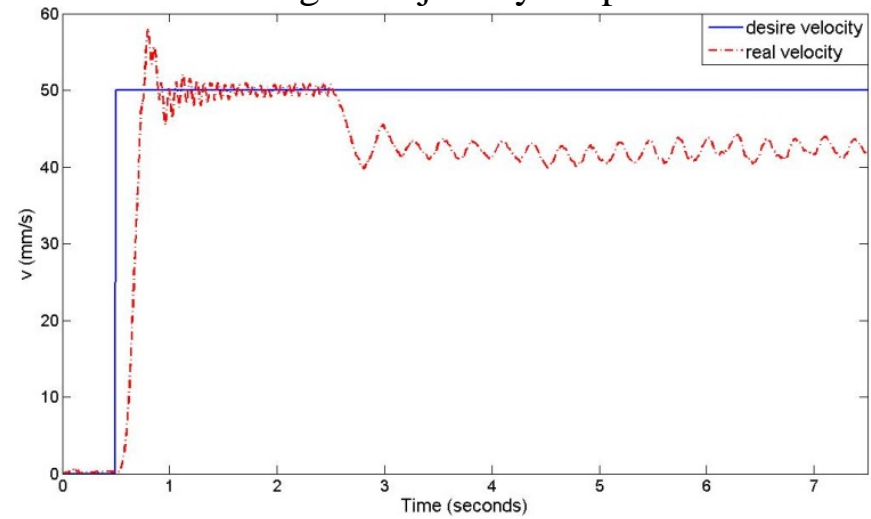

Fig.6 Longitudinal Velocity Tracking 


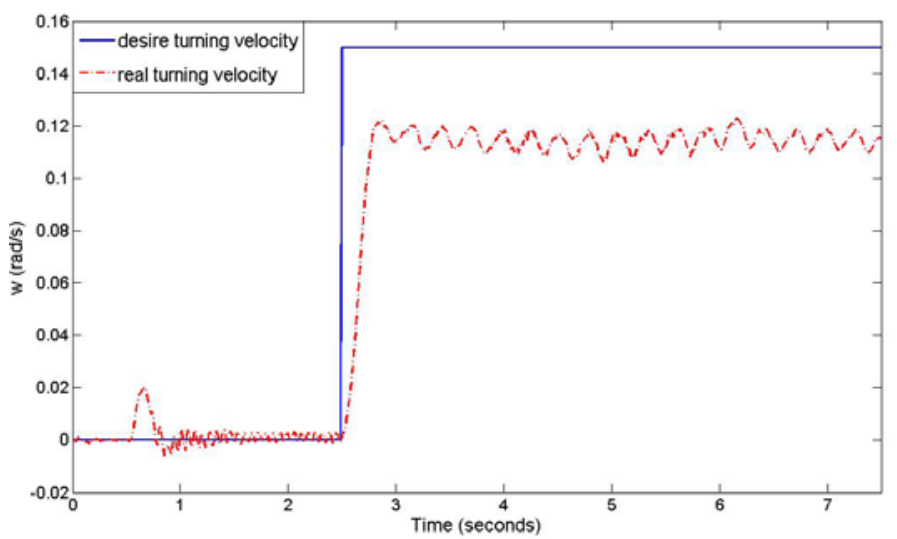

Fig.7 Steering Angle Velocity Tracking

Discussion: Fig.5 shows that the trajectory map reflects very well that the TMR virtual prototype is in accordance with the set of the control signal movement: from $0.5 \mathrm{~s}$ to $2.5 \mathrm{~s}$, the TMR is straight line movement, and then is turning movement.

Fig. 6 shows the longitudinal velocity tracking conforms to the control rules and $0.5 \mathrm{~s}-2.5 \mathrm{~s}$ in linear motion stage, virtual prototyping speed fluctuated in $50 \mathrm{~mm} / \mathrm{s}$ speed control; and after $2.5 \mathrm{~s}$, circular motion stage, longitudinal velocity value has a significant decline, and finally at a rate of $40 \mathrm{~mm} / \mathrm{s}$ fluctuated. Similarly, as can be seen from Fig.7, after the start of the circular motion, the speed of the virtual prototype does not reach the desired $0.15 \mathrm{rad} / \mathrm{s}$, but there is a static difference in the fluctuation of $0.11 \mathrm{rad} / \mathrm{s}$. The reason for this phenomenon lies in the speed distribution model in the control model. This is because the speed assignment model based on the simple kinematics model, which does not take into account the slip of the track. This makes the calculation of the track speed of the track wheel of the simple kinematics model is consumed by the slip of the track, and then, the longitudinal velocity and the steering speed of the robot are lower than the expected value.

\section{Summary}

In this paper, based on the multi-body dynamics software RecurDyn, the virtual prototype is firstly established of a tracked mobile robot. Then through the control interface provided by RecurDyn, connection control software Simulink, set up a joint control simulation platform of the tracked mobile robot. Based on tracked mobile robot longitudinal velocity and steering angular velocity tracking control simulation experiments verify the effectiveness of the combined control simulation platform, also found that the speed distribution model of simple kinematic model will make the vertical speed of the robot and the steering angular velocity tracking values are below expectations based on the proposed requirements for the improved speed distribution model, simulation research and control algorithm for the next movement laid the foundation.

\section{References}

[1] ZHOU Bo, Dai Xianzhong, HAN Jianda, Online Modelling and Tracking Control of Mobile Robots with Slippage in Outdoor Environments [J]. ROBOT,2011. 33(3): 265-272.

[2] LI Yan, et al. Dynamics Model and Feedback Control of Tracked Robots [J]. J Tsinghua Univ. (Sci. \& Tech). 2006, 46(8):1377-1380.

[3] LUO Guoqing, et al. Dynamic Simulation of a Tracked Vehicle Based on RecurDyn [J]. Vehicle \& Power Technology. 2011(04).

[4] SUN Fengchun, CHEN Shuyong. Matching Theory of Tracked Vehicle Induction Motor Drive System [J]. CHINESE JOURNAL OF MECHANICAL ENGINEERING. 2008, 44(11):260-266.

[5] LIU Yi , et al. Application and Improvement of RecurDyn, Multibody Dynamics Simulation [M]. 2013, Beijing: Electronic Industry Press.

[6] HUANG Tieqiu, et al. Integrated Simulation Modes Study of Dynamics and Control Based on RecurDyn [J]. Aerospace Control. 2010(03). 